\title{
Exosome Analysis in Tumor-Draining Pulmonary Vein Identifies NSCLC Patients with Higher Risk of Relapse after Curative Surgery
}

\author{
Alfons Navarro $\left.{ }^{1, *(}\right)$, Laureano Molins ${ }^{2}\left(\mathbb{D}\right.$, Ramon M. Marrades ${ }^{3}$, Jorge Moises ${ }^{3} \oplus$, \\ Nuria Viñolas ${ }^{4}$, Sara Morales ${ }^{1}{ }^{\circledR}$, Jordi Canals ${ }^{1}$, Joan J. Castellano ${ }^{1}$, José Ramírez ${ }^{5}$ \\ and Mariano Monzo ${ }^{1, *}$ \\ 1 Molecular Oncology and Embryology Laboratory, Human Anatomy Unit, School of Medicine, \\ University of Barcelona, IDIBAPS, 08036 Barcelona, Spain; saramolo.19@gmail.com (S.M.); \\ canals.serrat@ub.edu (J.C.); joan.castellano@ub.edu (J.J.C.) \\ 2 Department of Thoracic Surgery, Institut Clínic de Respiratori (ICR), Hospital Clínic de Barcelona, \\ University of Barcelona, 08036 Barcelona, Spain; 1molins@clinic.cat \\ 3 Department of Pneumology, Institut Clínic de Respiratori (ICR), Hospital Clínic de Barcelona, \\ University of Barcelona, IDIBAPS, CIBER de Enfermedades Respiratorias (CIBERES), 08036 Barcelona, \\ Spain; marrades@clinic.cat (R.M.M.); jrmoises@clinic.cat (J.M.) \\ 4 Department of Medical Oncology, Institut Clínic Malalties Hemato-Oncològiques (ICMHO), \\ Hospital Clínic de Barcelona, University of Barcelona, IDIBAPS, 08036 Barcelona, Spain; nvinolas@clinic.cat \\ 5 Department of Pathology, Centro de Diagnóstico Biomédico (CDB), Hospital Clínic de Barcelona, \\ University of Barcelona, IDIBAPS, CIBERES, 08036 Barcelona, Spain; jramirez@clinic.cat \\ * Correspondence: anavarroponz@ub.edu (A.N.); mmonzo@ub.edu (M.M.)
}

Received: 21 January 2019; Accepted: 18 February 2019; Published: 21 February 2019

\begin{abstract}
Since tumor-draining pulmonary vein blood (PV) is enriched in tumor-secreted products, we hypothesized that it would also be enriched in tumor-derived exosomes, which would be important in the metastasis process. We characterized exosomes from PV of 61 resected non-small cell lung cancer (NSCLC) patients to evaluate its potential as relapse biomarkers. Exosomes were characterized using transmission electron microscopy, western blot and nanoparticle tracking analysis and we examined time to relapse (TTR) and overall survival (OS). Differences between PV and peripheral vein were found. PV was enriched in smaller exosomes than the paired peripheral vein $(p=0.01)$. Moreover, PV exosome size mode was able to identify relapsed patients (Area under the curve $[\mathrm{AUC}]=0.781 ; 95 \% \mathrm{CI}$ : 0.6641-0.8978), in whom exosome size was smaller $(<112 \mathrm{~nm}$; $p<0.001$ ). The combination of PV exosome size and N (lymph node involvement) showed an AUC of 0.89 (95\%CI: 0.80-0.97). Moreover, smaller PV exosome size was associated with shorter TTR (28.3 months vs. not reached, $p<0.001$ ) and OS (43.9 months vs. not reached, $p=0.009$ ). Multivariate analyses identified PV exosome size and stage as independent prognostic markers for TTR and OS. PV exosome size is a promising relapse biomarker after surgery that can add valuable information to clinical variables.
\end{abstract}

Keywords: exosome; extracellular vesicles; tumor-draining vein; relapse; lung cancer; NSCLC

\section{Introduction}

Treatment of non-small cell lung cancer (NSCLC) depends on disease stage. In early and locally advanced stages, surgical resection of the tumor is, when possible, the first option and is considered curative [1]. In stage II and IIIa, adjuvant chemotherapy after surgery has been shown to improve survival [2]. However, the prognosis of these patients is still dismal-five-year survival rates of 
$60-80 \%$ for stage I, $20-50 \%$ for stage II and $23 \%$ for stage IIIA patients-due to high relapse rates $(40 \%)$ after surgery. One of the factors associated with patient relapse is the presence of circulating tumor cells (CTCs) in peripheral blood, which has been associated with worse prognosis [3]. However, studies in peripheral blood are limited by the low number of CTCs that can be detected, leading some investigators to analyze the utility of detecting CTCs and other biomarkers in blood from the tumor-draining pulmonary vein, which can better identify patients with a high risk of relapse after surgery [4]. While these results are interesting, the metastatic process cannot be explained only by the presence of CTCs, since their survival in the bloodstream, the extravasation process and the anchorage to the metastatic site are challenging. Recently, it has been shown that extracellular vesicles signals emitted by the primary tumor that are picked up by specific cells with the appropriate receptors [5], can contribute to the oncogenesis and metastasis process. The target cells can be located either in the local microenvironment of the tumor or in distant organs, since exosomes can reach the bloodstream. Extracellular vesicles are involved in several biological functions, including cross-talk between cells, tumorogenesis, drug resistance, angiogenesis promotion, and metastasis [6,7]. The extracellular vesicles are classified according to size and biogenesis as exosomes $(<150 \mathrm{~nm}$ and produced from multivesicular bodies) or microvesicles ( $>150 \mathrm{~nm}$ and formed through the direct budding of the plasma membrane) [7]. Exosomes have been shown to play a role in the metastatic process through the creation of a pre-metastatic niche that facilitates the anchorage and growth of CTCs in the metastatic site [8]. Recently, it has been also shown in vitro and in vivo in liver cancer that primary-tumor derived exosomes can deliver their cargo on CTCs and enhance their adhesion supporting metastasis formation [9].

To our knowledge, most of the studies have been focused on the study of exosome cargo to identify diagnosis/prognosis biomarkers, such as exosomal microRNAs [10] or exosomal proteins [11], and few studies have analyzed whether exosome levels themselves could be used as a disease parameter. Recently, König et al. have shown that the analysis of extracellular vesicles concentration in blood of breast cancer patients may serve as a complementary parameter reflecting the status of minimal residual disease as well as therapy and disease outcome in parallel with CTC investigation [12].

We hypothesized that the quantification and size distribution of exosomes in circulation could be an indicator of tumor metastatic capacity in non-small cell lung cancer. However, we speculate that tumor-derived exosomes in peripheral blood will be very diluted in comparison with tumor-draining pulmonary vein as previously observed with CTCs, especially in early-stage patients [13-15]. Therefore, we hypothesized that the analysis of tumor-derived exosomes could provide more reliable results in blood obtained from the pulmonary vein, where the products released by the tumor would be concentrated. We have compared the quantity and size of exosomes from tumor-draining pulmonary and paired peripheral vein and evaluated their potential as a biomarker of relapse in NSCLC.

\section{Results}

\subsection{Patients}

The analysis included 61 patients (Table 1). Twenty-three patients $(37.7 \%)$ received adjuvant cisplatin-based chemotherapy ( 2 stage IB, 16 stage II, and 5 stage III). None of the patients received neoadjuvant treatment. Median follow-up time was 40.87 months (IQR: 23.53-49.27). Twenty-one patients $(34.4 \%)$ relapsed after surgery, 15 with distant metastases. Seventeen patients $(27.8 \%)$ died during follow-up; although the cause of death was not recorded, $14(82.4 \%)$ of them died after relapse while three $(17.6 \%)$ died without relapse, suggesting that lung cancer was the main cause of death $(p<0.001)$. Pulmonary vein blood was obtained from the entire cohort, but blood from the peripheral vein was only obtained from 55 of the 61 patients $(92 \%)$. 
Table 1. Main clinical characteristics of the patients and univariate $p$-values (log-rank test) for time to relapse (TTR) and overall survival (OS).

\begin{tabular}{|c|c|c|c|c|}
\hline \multirow{2}{*}{ Characteristics } & \multirow{2}{*}{ Value } & $N=61$ & \multirow{2}{*}{$\begin{array}{c}\text { TTR } \\
p \text {-Value }\end{array}$} & \multirow{2}{*}{$\frac{\text { OS }}{p \text {-Value }}$} \\
\hline & & $N(\%)$ & & \\
\hline \multirow[t]{2}{*}{ Sex } & Male & $41(67.2)$ & & \\
\hline & Female & $20(32.8)$ & 0.5427 & 0.6291 \\
\hline \multirow[t]{3}{*}{ Age, $y$} & Mean (Range) & $63(32-80)$ & & \\
\hline & $\leq 65$ & $34(55.7)$ & & \\
\hline & $>65$ & $27(44.3)$ & 0.8 & 0.2253 \\
\hline \multirow[t]{2}{*}{ ECOG PS } & 0 & $26(42.6)$ & & \\
\hline & 1 & $35(57.4)$ & 0.4723 & 0.3479 \\
\hline \multirow[t]{3}{*}{ Pathological Stage } & I & $30(49.2)$ & & \\
\hline & II & $24(39.3)$ & & \\
\hline & III & $7(11.5)$ & 0.0069 & 0.0082 \\
\hline \multirow[t]{3}{*}{ Histology } & Adenocarcinoma & $35(57.4)$ & & \\
\hline & $\begin{array}{l}\text { Squamous cell } \\
\text { carcinoma }\end{array}$ & $16(26.2)$ & & \\
\hline & Others & $10(16.4)$ & 0.7562 & 0.9579 \\
\hline \multirow[t]{4}{*}{ Type of surgery } & Segmentectomy & $5(8.2)$ & & \\
\hline & Lobectomy/bilobectomy & 48 (78.7) & & \\
\hline & Pneumonectomy & $7(11.5)$ & & \\
\hline & Atypical Resection & $1(1.6)$ & 0.3963 & 0.4178 \\
\hline \multirow[t]{3}{*}{ Smoking history } & Current Smoker & $32(52.5)$ & & \\
\hline & Former Smoker & $24(39.3)$ & & \\
\hline & Never smoker & $5(8.2)$ & 0.1118 & 0.1881 \\
\hline \multirow[t]{2}{*}{$\mathrm{FEV}_{1}$} & Liters $( \pm \mathrm{SD})$ & $2.4( \pm 0.6)$ & & \\
\hline & $\%$ pred $( \pm \mathrm{SD})$ & $76.8( \pm 16.1)$ & - & - \\
\hline \multirow[t]{2}{*}{ FVC } & Liters $( \pm \mathrm{SD})$ & $3.56( \pm 0.74)$ & & \\
\hline & \%pred $( \pm \mathrm{SD})$ & $88( \pm 16.42)$ & - & - \\
\hline $\mathrm{FEV}_{1} / \mathrm{FVC}$ & Ratio $( \pm \mathrm{SD})$ & $67.2( \pm 9.74)$ & - & - \\
\hline Tumor size & $\mathrm{mm}( \pm \mathrm{SD})$ & $38.3( \pm 23.2)$ & - & - \\
\hline \multirow[t]{2}{*}{ Received adjuvant chemotherapy } & Yes & $23(37.7)$ & & \\
\hline & No & $38(62.3)$ & 0.0113 & 0.1958 \\
\hline \multirow[t]{2}{*}{ Experienced recurrence } & No & 40 (65.6) & & \\
\hline & Yes & $21(34.4)$ & - & - \\
\hline
\end{tabular}

ECOG PS, Eastern Cooperative Oncology Group performance status; $\mathrm{FEV}_{1}$, forced expiratory volume in $1 \mathrm{~s}$; FVC, forced vital capacity. Bold for $p<0.05$.

\subsection{Exosome Characterization}

Transmission electron microscopy (TEM) morphological analysis showed the presence of round 27-200 $\mathrm{nm}$ vesicles. To further validate that these vesicles were, at least in part, exosomes, the presence of the established exosomal markers TSG101 and CD63 was determined using western blot, which confirmed the presence of the exosomes (Figure 1A). Using nanoparticle tracking analysis (NTA), which uses the properties of both Brownian motion and light scattering to obtain the particle size distribution of samples in a liquid suspension (Video S1), we obtained a size distribution profile graph (Figure 1B), and concentration (nanoparticles $/ \mathrm{mL}$ ) and size measures for each sample. The median concentration in all samples by NTA was $3.815 \times 10^{9}$ particles per $\mathrm{mL}$ (range: $7.72 \times 10^{8}-2.64 \times 10^{10}$ ) and the mode of particle diameter was $114.5 \mathrm{~nm}$ (range: 85.1-173.3).

\subsection{Exosome Concentration and Clinical Characteristics}

When we compared the overall exosome concentration between the pulmonary and peripheral veins, no significant differences were observed ( $p=0.1214$, Figure 1C). However, the sub-analysis of the specific levels of different exosome sizes showed that the pulmonary vein was enriched in 
exosomes of 30-50 nm ( $p=0.0496)$ while no significant differences were observed for other group sizes (51-150/151-1000 nm, Figure 1D).

The correlation of the overall exosome concentration with the main clinical characteristics of the patients showed that the quantity of exosomes in the pulmonary vein was related to the $\mathrm{T}$ stage (of the TNM classification), where T1 patients had significantly fewer exosomes than others $(p=0.0204$, Figure 1E). However, this association was not observed in the paired blood from the peripheral vein ( $p=0.6251$, Figure 1F). Moreover, when we grouped patients according to median tumor size, those with tumors smaller than $35 \mathrm{~mm}$ had fewer pulmonary vein exosomes than those with larger tumors $(p=0.01)$. No significant association was observed in the peripheral vein.

A

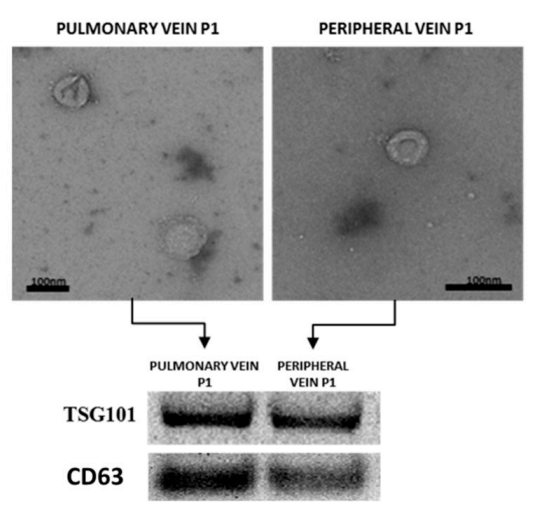

C

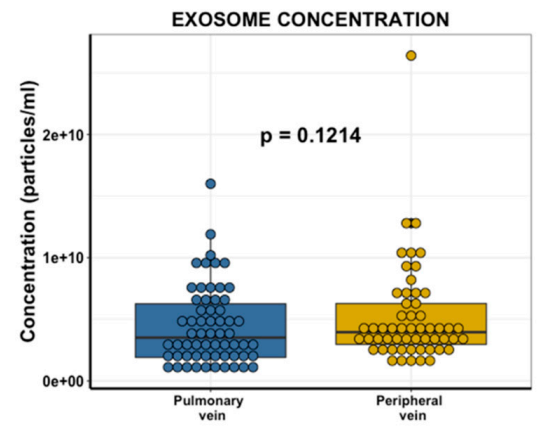

E

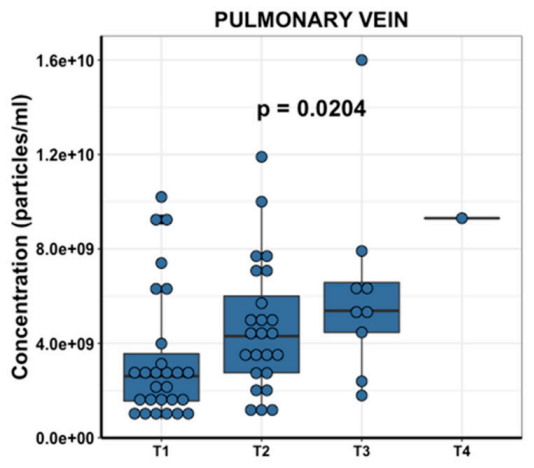

B

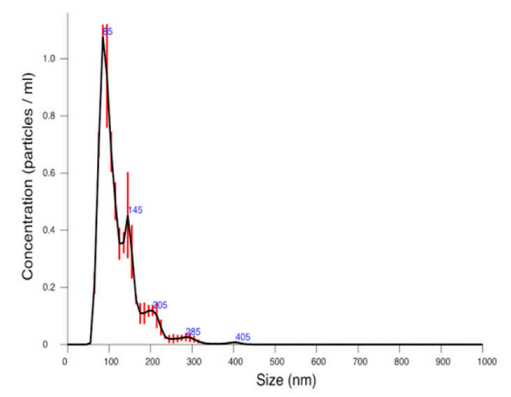

D

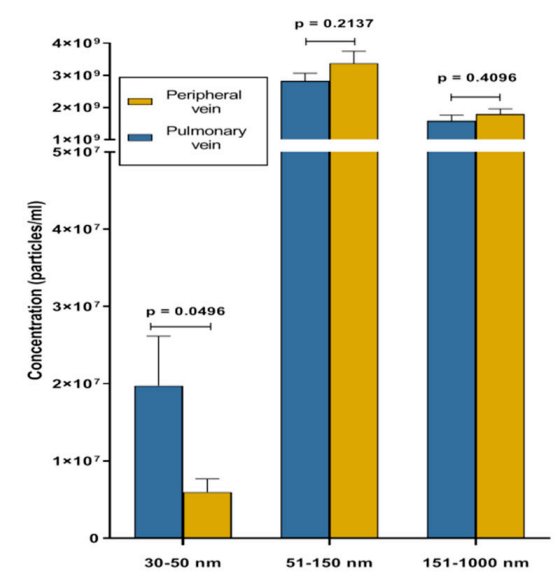

$\mathbf{F}$

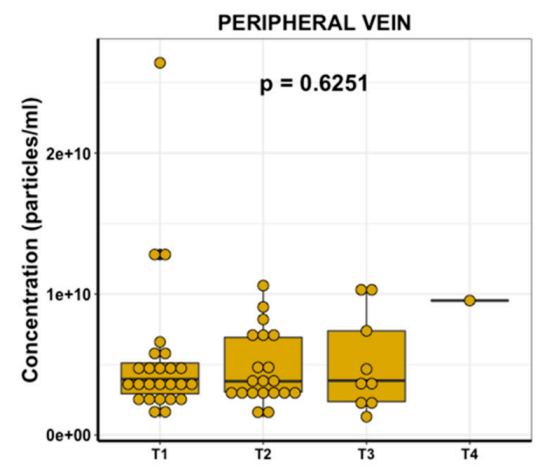

Figure 1. (A) Exosomes were characterized by transmission electron microscopy using negative staining and Western blot using the exosome marker TSG101. (B) The quantification and size analysis were performed by Nanosight. An example of the size distribution graph obtained is shown. (C) Boxplot showing exosome levels in pulmonary and peripheral vein in non-small cell lung cancer (NSCLC) patients. (D) Bar plot showing exosomal size levels in pulmonary and peripheral vein according to three size groups: 30-50 nm, 51-150 nm and 151-1000 nm. (E) Boxplot showing pulmonary vein exosome levels according to T stage in NSCLC patients. (F) Boxplot showing peripheral vein exosome levels according to T stage in NSCLC patients. 


\subsection{Pulmonary Vein Exosome Size Identifies Patients Who Relapse after Curative Surgery}

Significant differences in exosome size between pulmonary and peripheral veins were observed (Figure 2A). Pulmonary vein exosomes were significantly smaller than those from the paired peripheral vein (mean mode size 111.8754 vs. $118.1545 ; p=0.0134$ ).

The size of exosomes from the pulmonary vein was significantly smaller in relapsed patients than in non-relapsed patients (mean size 105.26 vs. $115.35 \mathrm{~nm}, p<0.001$ ) (Figure 2B). Nevertheless, these differences in size were not observed in the peripheral vein $(p=0.62$, Figure $2 \mathrm{C})$.

ROC curves were generated to investigate exosome size as a predictive biomarker of relapse after surgery. The area under the curve (AUC) value according to pulmonary vein exosome size was 0.78 (95\% confidence interval (CI), $0.6641-0.8978)$ with a sensitivity of $67.5 \%$ and specificity of $81 \%$ in distinguishing patients who relapse after surgery in its best threshold (112 nm) (Figure 2D). Using $112 \mathrm{~nm}$ as a threshold, the negative predictive value was $87.1 \%$, and the positive predictive value was $56.7 \%$.

In order to compare the impact of the pulmonary vein exosome size with other clinical factors, lymph node involvement $(\mathrm{N})$, and disease stage were included in the analysis. $\mathrm{N}$ showed an AUC of 0.76 (95\% CI: 0.64-0.88), and disease stage showed an AUC of 0.71 (95\% CI, $0.59-0.84)$. To verify that no other confounding parameters could have affected these results, we performed a binary logistic regression multivariate analysis for relapse to verify the independent impact of pulmonary vein exosome size to predict relapse after curative surgery. Pulmonary vein exosome size (hazard ratio (HR), 0.87 (95\%CI, 0.79-0.94); $p=0.0019)$ and N (HR, 7.1 (95\% CI, 2.48-27.14); $p=0.0011)$ emerged as independent markers of relapse. Interestingly, when we combined pulmonary vein exosome size and $\mathrm{N}$, a good AUC of 0.89 (95\% CI, 0.80-0.97) was obtained.

A

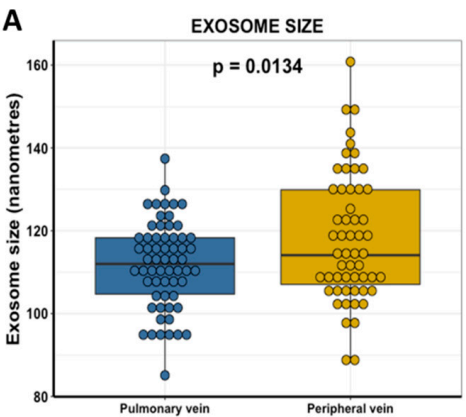

C

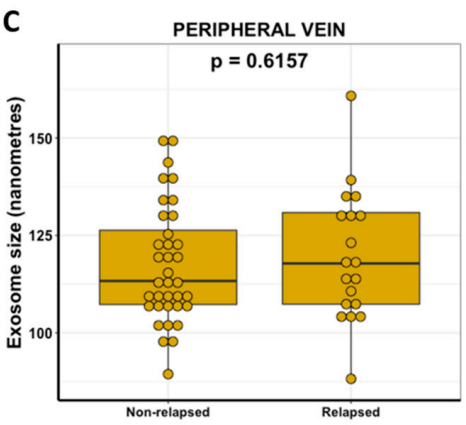

B

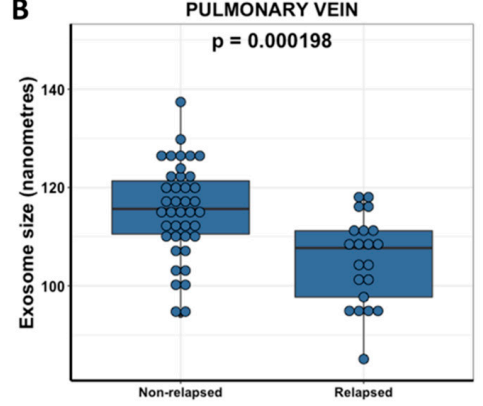

D

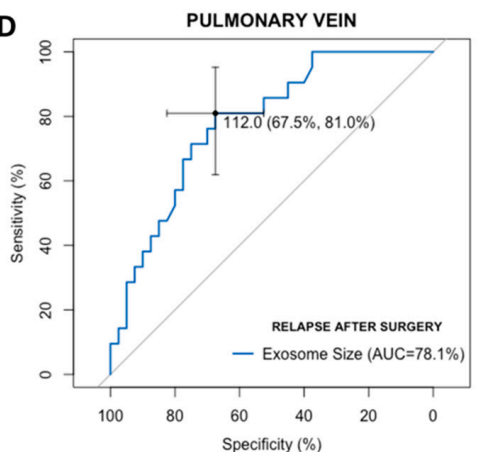

Figure 2. The relationship between exosomal size and relapse after surgery in NSCLC patients. (A) Boxplot showing exosome size distribution (size mode) in pulmonary and peripheral vein in NSCLC patients. (B) Boxplot showing pulmonary vein exosomal size in relapsed and non-relapsed NSCLC patients. (C) Boxplot showing peripheral vein exosomal size in relapsed and non-relapsed NSCLC patients. (D) ROC curve analysis of pulmonary vein exosomal size values predicting relapse after surgery in NSCLC patients. The cut-off for disease relapse after surgery was set at $112 \mathrm{~nm}$. The area under the curve of 0.78 with pulmonary vein exosomal size values of $<112 \mathrm{~nm}$ resulted in a sensitivity of $81 \%$ and specificity of $67.5 \%$. 


\subsection{Pulmonary Vein Exosome Size Is Associated with Outcome after Curative Surgery}

The smaller exosome size $(<112 \mathrm{~nm})$ in the pulmonary vein was associated with shorter TTR (28.3 months vs. not reached; $p=0.0004$; Figure 3A) and shorter OS (43.9 months vs. not reached; $p=0.0092$; Figure 3B). No significant differences were observed when exosome size was analyzed in the peripheral vein (Figure 3C,D).

A
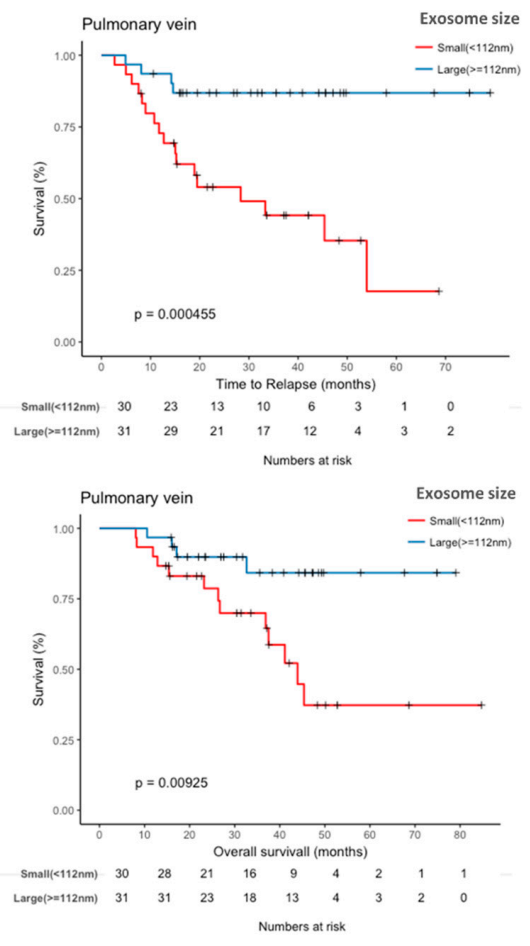

C

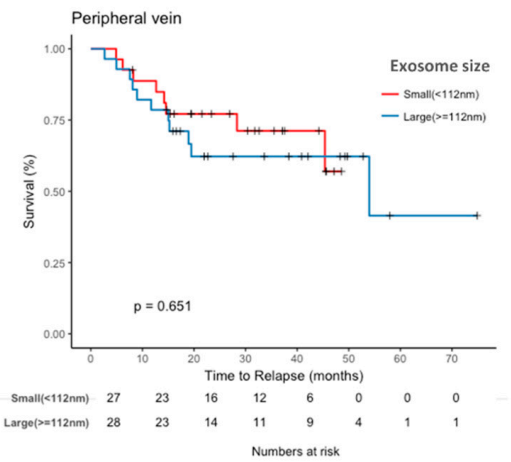

D

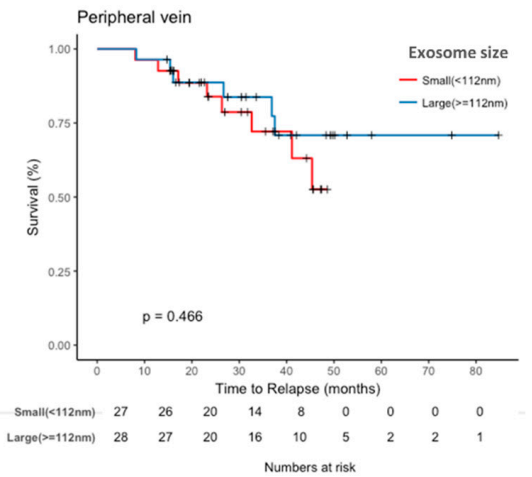

Figure 3. Kaplan-Meier survival analysis of associations between exosomal size and time to relapse (TTR) and overall survival (OS) among patients with NSCLC. (A) TTR according to pulmonary vein exosomal size. (B) OS according to pulmonary vein exosomal size. (C) TTR according to peripheral vein exosomal size. (D) OS according to peripheral vein exosomal size.

An exploratory analysis of patients with stage I disease $(n=30)$ showed that exosome size discriminated stage I patients with a high relapse risk (AUC=0.76 (95\% CI, 0.53-0.98)) (Figure 4A). In addition, a trend towards shorter TTR $(p=0.0763)$ and OS $(p=0.1190)$ was observed for patients with smaller exosome size (Figure 4B,C).

A

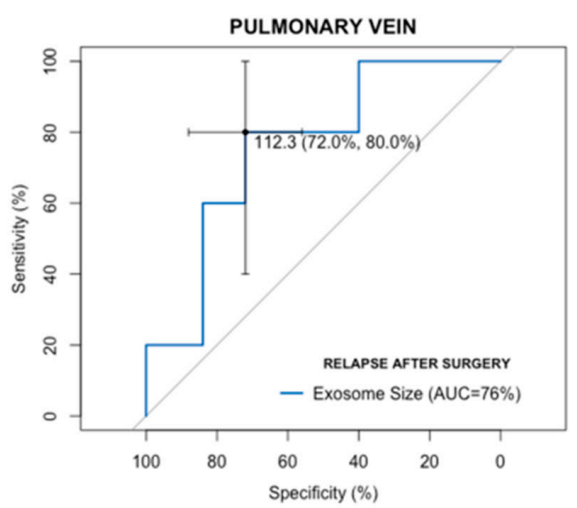

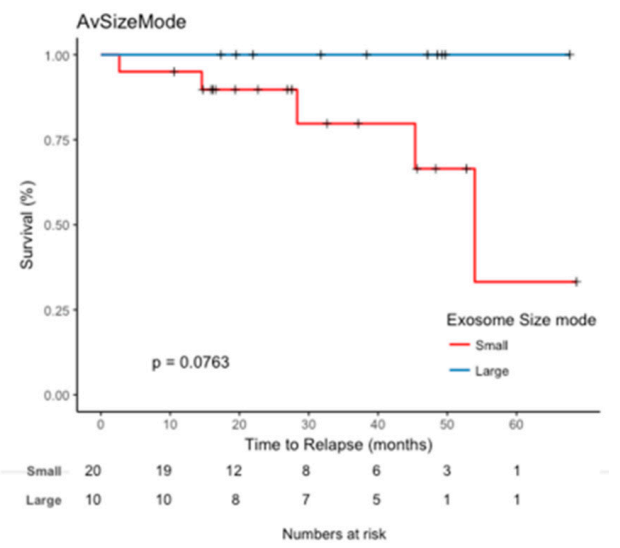

Figure 4. Cont. 


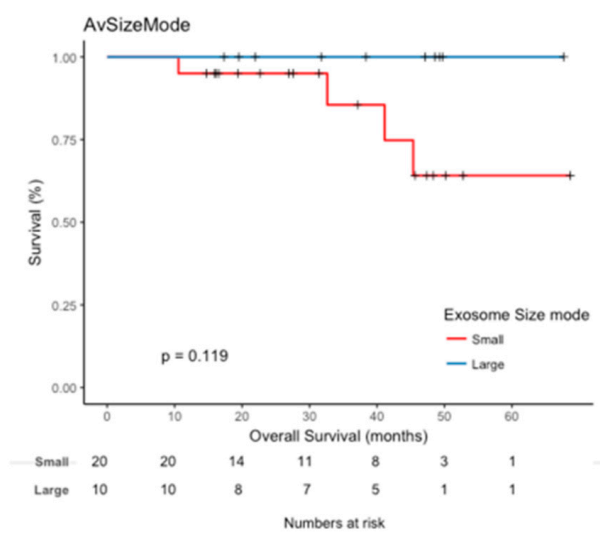

Figure 4. (A) ROC curve analysis of pulmonary vein exosomal size values predicting relapse after surgery in stage I NSCLC patients. The cut-off for relapse was set at $112.3 \mathrm{~nm}$. The area under the curve of 0.76 with pulmonary vein exosomal size values of $<112.3 \mathrm{~nm}$ resulted in a sensitivity of $80 \%$ and specificity of $72 \%$. (B) Kaplan-Meier survival analysis of associations between pulmonary vein exosomal size and time to relapse (TTR) among patients with stage I NSCLC. (C) Kaplan-Meier survival analysis of the association between pulmonary vein exosomal size and overall survival (OS) among patients with stage I NSCLC.

\subsection{Cox Modeling of Relapse and Survival}

In the multivariate Cox analyses, exosome size emerged as an independent risk factor for TTR (HR, 6.66 (95\%CI, 2.06-21.51); $p=0.0015)$ and OS (HR, $4.55(95 \% \mathrm{CI}, 1.43-14.49) ; p=0.0104)$. Stage was also an independent marker of TTR (HR, 2.42 (95\% CI, 1.16-5.03); $p=0.0180)$ and OS (HR, 2.93 (95\% CI, $1.46-5.84) ; p=0.0024)$ (Table 2).

Table 2. Cox multivariate analyses of time to relapse and overall survival. Bold for $p<0.05$.

\begin{tabular}{lcc}
\hline \multicolumn{1}{c}{ Time to Relapse } & Hazard Ratio (HR, 5\% CI) & $\boldsymbol{p}$-Value \\
\hline Stage & $2.42(1.16-5.03)$ & $\mathbf{0 . 0 1 8 0}$ \\
Adjuvant chemotherapy & $1.59(0.59-4.26)$ & 0.3563 \\
Exosome size in pulmonary vein $<112 \mathrm{~nm}$ & $6.66(2.06-21.51)$ & $\mathbf{0 . 0 0 1 5}$ \\
\hline Overall Survival & HR (95\% CI) & $p$-value \\
\hline Stage & $2.93(1.46-5.84)$ & $\mathbf{0 . 0 0 2 4}$ \\
Exosome size in pulmonary vein $<112 \mathrm{~nm}$ & $4.55(1.43-14.49)$ & $\mathbf{0 . 0 1 0 4}$ \\
\hline
\end{tabular}

\section{Discussion}

In the present study, we have examined exosome size distribution and levels to evaluate their potential to predict relapse of resected NSCLC patients. Importantly, exosomes were analyzed in blood obtained from the pulmonary vein during surgery and compared with those obtained from peripheral blood before tumor resection. Previous studies showed that blood obtained from a tumor-draining vein is enriched in tumor-secreted products, including CTCs [13], which led us to hypothesize that it would be enriched in primary tumor-derived exosomes. Our results showed that pulmonary vein blood was enriched in exosomes of 30-50 nm, while no differences were observed in other size ranges. Remarkably, pulmonary vein overall exosome levels were more highly correlated with tumor characteristics than those in peripheral blood. In this line, we observed a significant association between $\mathrm{T}$ stage and pulmonary vein — but not peripheral vein-exosome levels. T stage is related to tumor size and we observed higher pulmonary vein exosome levels in tumors larger than the median $(35 \mathrm{~mm})$. Pulmonary vein exosome analysis may more closely represent tumor characteristics than the analysis of peripheral vein. 
Moreover, significant differences in exosome size distribution (mode of size) were observed between the pulmonary and peripheral veins, where pulmonary vein exosomes were smaller than peripheral vein exosomes. Interestingly, the size of pulmonary vein exosomes correlated with the risk of post-surgical relapse and survival. Patients with smaller pulmonary vein exosomes had a higher risk of relapse and shorter TTR and OS, while no differences were observed according to peripheral vein exosome size. In line with our results, an in vitro study in prostate cancer found that the amount and size of exosomes changed after the acquisition of resistance to docetaxel treatment, where the size of exosomes secreted by resistant cells was smaller than of those secreted by the sensitive cells [5]. Recently, Kim et al. reported that extracellular vesicle size was an independent predictor of progression-free survival at first response assessment to chemotherapy in pancreatic cancer [16]. Taken together with our results, these findings allow us to speculate that a more aggressive cell phenotype is linked to the production of smaller exosomes.

The evaluation of pulmonary vein exosome size was able to identify patients who relapsed with an accuracy of $72 \%$. The AUC of 0.78 for exosome size observed in our cohort is fair and was able to discriminate relapsed patients more accurately than disease stage (AUC of 0.71), or N (AUC of 0.76). Interestingly, the good AUC of 0.89 for the combination of exosome size and $\mathrm{N}$ suggest that exosome size may potentially complement clinical variables in identifying patients with a high risk of relapse, who could benefit from more aggressive treatment. This is especially relevant for stage I patients, who do not receive any post-surgical treatment, in comparison with stage II-IIIa patients who benefit from platinum-based chemotherapy [1]. In a sub-analysis of stage I patients, exosome size as a continuous variable also discriminated patients with a high relapse risk and shorter TTR (Cox univariate HR, 0.91 (95\% CI, 0.829-0.9998); $p=0.0496$ ). These preliminary results, if confirmed in larger studies, indicate that pulmonary vein exosome size is a promising biomarker of relapse for stage I patients.

Despite the benefits described in our work and others [13-15] of the analysis of tumor-secreted products in tumor-draining pulmonary vein, the obtaining of this type of sample could be considered invasive in comparison with peripheral blood analysis. However, we have to take into account that this analysis is limited to surgical NSCLC patients, where the access to the pulmonary vein is neither modifying the routine surgical procedure nor requiring additional lung dissection. The tumor-draining pulmonary vein is punctured immediately on opening the chest prior to beginning the resection of the tumor. This approach was safe with no complications reported in our cohort of patients or in previous studies [13]. Moreover, most of the resected NSCLC patients are early-stage (I-II) patients in which the number of tumor-secreted products in peripheral blood is more limited, and, therefore, they will be the most benefited from the tumor-draining pulmonary vein analysis.

Several authors have found that exosomes and their cargo, analyzed in peripheral blood $[11,17,18]$ and bronchoalveolar lavage [19], can be useful diagnostic biomarkers in NSCLC. However, to our knowledge, this is the first report to analyze exosomes in blood from the pulmonary vein and to show that they can be used as an independent prognostic biomarker for relapse and survival after surgery in NSCLC. Further investigation in a larger cohort of patients is warranted to validate these findings and explore the potential for exosome size analysis to be used in the clinical management of resected NSCLC patients.

\section{Material and Methods}

\subsection{Patient Samples}

The study included 61 stage I-IIIa NSCLC patients who underwent complete surgical resection in our institution (Table 1). Blood (4 mL in EDTA tube) from the tumor-draining pulmonary vein was obtained during the surgery before tumor resection or vessel ligation. Blood from a peripheral vein was obtained by the nurse anesthesiologist before the start of surgery. Plasma obtained by centrifugation was stored at $-80^{\circ} \mathrm{C}$ until processing. Written informed consent was obtained from each participant 
in accordance with the Declaration of Helsinki and the study was approved by the Clinical Research Ethics Committee of the Hospital Clínic de Barcelona (project approval number HCB/2017/1052).

\subsection{Exosome Purification and Characterization}

Exosomes were isolated from $200 \mu \mathrm{L}$ of plasma by ultracentrifugation in a Sorvall MX Plus Micro-Ultracentrifuge with S140AT Rotor as previously described [20]. Pelleted exosomes resuspended in $200 \mu \mathrm{L}$ of dPBS: $100 \mu \mathrm{L}$ were used for exosome characterization by TEM and Western blot and $100 \mu \mathrm{L}$ were used for exosome quantification and size analysis by NTA. Exosomal marker TSG101 was analyzed by Western blot as previously described [20,21], and CD63 was analyzed using Rabbit polyclonal to CD63 (ab68418, from Abcam, Cambridge, UK). For TEM analysis, exosomes in dPBS were fixed in a final concentration of $2 \%$ paraformaldehyde, mounted on copper-mesh formvar grids and negatively stained by $2 \%$ uranyl acetate. Samples were observed using a TEM JEOL J1010 80 kV at the Electron Cryomicroscopy Unit of the University of Barcelona (CCiTUB, Barcelona, Spain). NTA analysis was performed on a Nanosight NS300 in the ICTS "NANBIOSIS" (Biomaterial Processing and Nanostructuring Unit of the CIBER in Bioengineering, Biomaterials \& Nanomedicine at Institut de Ciència de Materials de Barcelona, CSIC, Campus UAB, Bellaterra, Barcelona, Spain).

\subsection{Statistical Analysis}

Assumptions of distributional normality were tested using the Shapiro-Wilk test and quantilequantile plot. Continuous data were tested with the T-test (two groups) or ANOVA (more than two groups) when normally distributed and the Mann-Whitney U test or Kruskal Wallis test when not normally distributed. Receiver operating characteristic (ROC) curves were calculated using R package pROC. Time to relapse (TTR) was calculated as the time between resection and recurrence or last follow-up and overall survival (OS) as the time between resection and death from any cause or last follow-up. Kaplan-Meier curves for TTR and OS were drawn and compared by means of a log-rank test. All clinical factors with $p<0.1$ in the univariate analysis were included in the Cox multivariate regression analyses. Statistical significance was set at $p \leq 0.05$. All statistical analyses were performed using R v3.3 (R Foundation for Statistical Computing, https:/ / www.r-project.org/).

\section{Conclusions}

The evaluation of tumor-draining pulmonary vein exosome size is a promising prognostic biomarker for relapse and survival after surgery and can add valuable information to clinical variables.

Supplementary Materials: The following are available online at http:/ / www.mdpi.com/2072-6694/11/2/249/s1, Video S1. Video captured during the nanoparticle tracking analysis (NTA) measurement in Nanosight NS300 equipment showing the movement of particles (exosomes) in suspension.

Author Contributions: A.N. had full access to all of the data in the study and takes responsibility for the integrity of the data, the accuracy of the data analysis and wrote the manuscript draft. L.M. was involved in the obtention of the tumor draining vein. L.M., R.M.M., J.M., N.V. and J.R. analyzed clinical data. S.M., J.C., J.J.C., performed research: M.M. contributed substantially to the study design and the writing of the manuscript. All authors read and approved the final version of the manuscript.

Acknowledgments: This work was supported by grants from AECC-Catalunya 2017 (A.N.), SAF2017-88606-P (A.N.) from the Ministry of Economy and Competition (MINECO) co-financed with the European Union FEDER funds, and SDCSD from the Universitat de Barcelona (M.M.). J.C. and J.J.C. are APIF fellows of the Universitat de Barcelona. Thanks to the "Ajuts de la Universitat de Barcelona per publicar en accés obert" for covering part of the the open access costs of this publication.

Conflicts of Interest: The authors declare no conflict of interest. The funders had no role in the design of the study; in the collection, analyses, or interpretation of data; in the writing of the manuscript, or in the decision to publish the results. 


\section{References}

1. Vansteenkiste, J.; De Ruysscher, D.; Eberhardt, W.; Lim, E.; Senan, S.; Felip, E.; Peters, S.; Group, E.G.W. Early and locally advanced non-small-cell lung cancer (NSCLC): ESMO Clinical Practice Guidelines for diagnosis, treatment and follow-up. Ann. Oncol. 2013, 24, vi89-vi98. [CrossRef] [PubMed]

2. Douillard, J.-Y.; Rosell, R.; De Lena, M.; Carpagnano, F.; Ramlau, R.; Gonzáles-Larriba, J.L.; Grodzki, T.; Pereira, J.R.; Le Groumellec, A.; Lorusso, V. Adjuvant vinorelbine plus cisplatin versus observation in patients with completely resected stage IB-IIIA non-small-cell lung cancer (Adjuvant Navelbine International Trialist Association [ANITA]): A randomised controlled trial. Lancet Oncol. 2006, 7, 719-727. [CrossRef]

3. Krebs, M.G.; Sloane, R.; Priest, L.; Lancashire, L.; Hou, J.-M.; Greystoke, A.; Ward, T.H.; Ferraldeschi, R.; Hughes, A.; Clack, G. Evaluation and prognostic significance of circulating tumor cells in patients with non-small-cell lung cancer. J. Clin. Oncol. 2011, 29, 1556-1563. [CrossRef] [PubMed]

4. Sienel, W.; Seen-Hibler, R.; Mutschler, W.; Pantel, K.; Passlick, B. Tumour cells in the tumour draining vein of patients with non-small cell lung cancer: Detection rate and clinical significance. Eur. J. Cardio-Thoracic Surg. 2003, 23, 451-456. [CrossRef]

5. van Niel, G.; D'Angelo, G.; Raposo, G. Shedding light on the cell biology of extracellular vesicles. Nat. Rev. Mol. Cell Biol. 2018, 19, 213. [CrossRef] [PubMed]

6. Shah, R.; Patel, T.; Freedman, J.E. Circulating Extracellular Vesicles in Human Disease. N. Engl. J. Med. 2018, 379, 958-966. [CrossRef] [PubMed]

7. Xu, R.; Rai, A.; Chen, M.; Suwakulsiri, W.; Greening, D.W.; Simpson, R.J. Extracellular vesicles in cancerImplications for future improvements in cancer care. Nat. Rev. Clin. Oncol. 2018, 15, 617-638. [CrossRef] [PubMed]

8. Peinado, H.; Alečković, M.; Lavotshkin, S.; Matei, I.; Costa-Silva, B.; Moreno-Bueno, G.; Hergueta-Redondo, M.; Williams, C.; García-Santos, G.; Ghajar, C.M. Melanoma exosomes educate bone marrow progenitor cells toward a pro-metastatic phenotype through MET. Nat. Med. 2012, 18, 883. [CrossRef] [PubMed]

9. Fu, Q.; Zhang, Q.; Lou, Y.; Yang, J.; Nie, G.; Chen, Q.; Chen, Y.; Zhang, J.; Wang, J.; Wei, T. Primary tumor-derived exosomes facilitate metastasis by regulating adhesion of circulating tumor cells via SMAD3 in liver cancer. Oncogene 2018, 37, 6105-6118. [CrossRef] [PubMed]

10. Munagala, R.; Aqil, F.; Gupta, R.C. Exosomal miRNAs as biomarkers of recurrent lung cancer. Tumor Biol. 2016, 37, 10703-10714. [CrossRef] [PubMed]

11. Sandfeld-Paulsen, B.; Aggerholm-Pedersen, N.; Baek, R.; Jakobsen, K.; Meldgaard, P.; Folkersen, B.; Rasmussen, T.R.; Varming, K.; Jørgensen, M.; Sorensen, B. Exosomal proteins as prognostic biomarkers in non-small cell lung cancer. Mol. Oncol. 2016, 10, 1595-1602. [CrossRef] [PubMed]

12. König, L.; Kasimir-Bauer, S.; Bittner, A.-K.; Hoffmann, O.; Wagner, B.; Santos Manvailer, L.F.; Kimmig, R.; Horn, P.A.; Rebmann, V. Elevated levels of extracellular vesicles are associated with therapy failure and disease progression in breast cancer patients undergoing neoadjuvant chemotherapy. Oncoimmunology 2018, 7, e1376153. [CrossRef] [PubMed]

13. Crosbie, P.A.; Shah, R.; Krysiak, P.; Zhou, C.; Morris, K.; Tugwood, J.; Booton, R.; Blackhall, F.; Dive, C. Circulating tumor cells detected in the tumor-draining pulmonary vein are associated with disease recurrence after surgical resection of NSCLC. J. Thoracic Oncol. 2016, 11, 1793-1797. [CrossRef] [PubMed]

14. Murlidhar, V.; Reddy, R.M.; Fouladdel, S.; Zhao, L.; Ishikawa, M.K.; Grabauskiene, S.; Zhang, Z.; Lin, J.; Chang, A.C.; Carrott, P. Poor prognosis indicated by venous circulating tumor cell clusters in early-stage lung cancers. Cancer Res. 2017, 77, 5194-5206. [CrossRef] [PubMed]

15. Reddy, R.M.; Murlidhar, V.; Zhao, L.; Grabauskiene, S.; Zhang, Z.; Ramnath, N.; Lin, J.; Chang, A.C.; Carrott, P.; Lynch, W. Pulmonary venous blood sampling significantly increases the yield of circulating tumor cells in early-stage lung cancer. J. Thoracic Cardiovasc. Surg. 2016, 151, 852-858. [CrossRef]

16. Kim, D.U.; San Lucas, F.A.; Cagley, M.; Stephens, B.; Mulu, F.C.; Castillo, J.; Bernard, V.; Varadhachary, G.R.; Katz, M.H.; Alvarez, H.A. Larger size extracellular vesicle as early biomarker in patients with metastatic pancreatic cancer. J. Clin. Oncol. 2017, 35, 234. [CrossRef]

17. Rabinowits, G.; Gerçel-Taylor, C.; Day, J.M.; Taylor, D.D.; Kloecker, G.H. Exosomal microRNA: A diagnostic marker for lung cancer. Clin. Lung Cancer 2009, 10, 42-46. [CrossRef]

18. Rahman, M.A.; Barger, J.F.; Lovat, F.; Gao, M.; Otterson, G.A.; Nana-Sinkam, P. Lung cancer exosomes as drivers of epithelial mesenchymal transition. Oncotarget 2016, 7, 54852. [CrossRef] 
19. Rodríguez, M.; Silva, J.; López-Alfonso, A.; López-Muñiz, M.B.; Peña, C.; Domínguez, G.; García, J.M.; López-Gónzalez, A.; Méndez, M.; Provencio, M. Different exosome cargo from plasma/bronchoalveolar lavage in non-small-cell lung cancer. Genes Chromosomes Cancer 2014, 53, 713-724. [CrossRef]

20. Monzo, M.; Santasusagna, S.; Moreno, I.; Martinez, F.; Hernáez, R.; Muñoz, C.; Castellano, J.J.; Moreno, J.; Navarro, A. Exosomal microRNAs isolated from plasma of mesenteric veins linked to liver metastases in resected patients with colon cancer. Oncotarget 2017, 8, 30859. [CrossRef]

21. Ruiz-Martinez, M.; Navarro, A.; Marrades, R.M.; Viñolas, N.; Santasusagna, S.; Muñoz, C.; Ramírez, J.; Molins, L.; Monzo, M. YKT6 expression, exosome release, and survival in non-small cell lung cancer. Oncotarget 2016, 7, 51515. [CrossRef] [PubMed]

2019 by the authors. Licensee MDPI, Basel, Switzerland. This article is an open access article distributed under the terms and conditions of the Creative Commons Attribution (CC BY) license (http://creativecommons.org/licenses/by/4.0/). 